Meta

Journal des traducteurs

Translators' Journal

\title{
L'argumentation concessive française et espagnole : le transfert en espagnol de quelques connecteurs (pourtant, quand même, même si, or...) et d'autres formules concessives
}

\section{Mercedes Tricás}

Volume 35, numéro 3, septembre 1990

La traduction dans le monde hispanolusophone

URI : https://id.erudit.org/iderudit/004509ar

DOI : https://doi.org/10.7202/004509ar

Aller au sommaire du numéro

Éditeur(s)

Les Presses de l'Université de Montréal

ISSN

0026-0452 (imprimé)

1492-1421 (numérique)

Découvrir la revue

Citer cet article

Tricás, M. (1990). L’argumentation concessive française et espagnole : le transfert en espagnol de quelques connecteurs (pourtant, quand même, même si, or...) et d'autres formules concessives. Meta, 35(3), 529-537.

https://doi.org/10.7202/004509ar d'utilisation que vous pouvez consulter en ligne. 


\title{
L'ARGUMENTATION CONCESSIVE FRANCAISE ET ESPAGNOLE : LE TRANSFERT EN ESPAGNOL DE QUELQUES CONNECTEURS (POURTANT, QUAND MEME, MÊME SI, OR...) ET D'AUTRES FORMULES CONCESSIVES
}

\author{
MERCEDES TRICÁS \\ Escuela de Traductores e Intérpretes, Universidad Autónoma de \\ Barcelona, España
}

Les études menées à terme, dans ces derniers temps, concernant les connecteurs notamment les travaux d'O. Ducrot, J.-C. Anscombre et de ce qu'on appelle déjà «l'École de Genève» d'Eddy Roulet - permettent d'envisager la sémantique de ces morphèmes d'une façon beaucoup plus approfondie.

Les traducteurs, préoccupés par les difficultés de transfert des noyaux lexicaux ou par les exigences d'adaptation de la structure syntaxique à la langue d'arrivée, ont très souvent méprisé l'importance des connecteurs. La traduction de ces mots est réalisée, dans la plupart des cas, d'une façon presque automatique en faisant appel aux connecteurs les plus généraux et les plus fréquents de la langue d'arrivée, et très rarement les traducteurs font l'effort de pénétrer dans les nuances argumentatives véhiculées par les différents connecteurs, ce qui permettrait un transfert beaucoup plus précis. Les traductions perdent ainsi une grande partie de l'expressivité de ces mots et les argumentations s'appauvrissent dans le passage d'une langue à l'autre. Il faut dire aussi que les dictionnaires — unilingues ou bilingues — n'aident pas beaucoup les traducteurs quand il s'agit de rendre la fidélité de ces morphèmes. Un bon dictionnaire de connecteurs serait un bon instrument de travail dans l'activité traductrice.

Notre but ici n'est que de montrer, d'une part, quelques connecteurs de concession français qui véhiculent des nuances sémantiques importantes négligées dans la plupart des traductions espagnoles et, d'autre part, quelques divergences générales dans la formulation de l'opération concessive en français et en espagnol. La limite de ces pages ne nous permet que de signaler les lignes fondamentales de ce problème. Nous allons aussi nous limiter aux textes écrits, le domaine le plus fréquent d'un traducteur, en laissant de côté les multiples variations sémantiques des connecteurrs dans l'interaction orale.

Nous avons choisi la concession à cause de la fréquence d'emploi de ces opérations argumentatives et aussi à cause de leur importance dans la cohérence textuelle. Nous envisageons le terme «concession» dans les deux sens généralement acceptés:

a) les concessions dites argumentatives, qui correspondent au schéma argumentatif du mais d'O. Ducrot ${ }^{1}$ :

- «p mc q» (où mc est le marqueur de concession) véhicule un argument $p$ qui devait mener à une conclusion $r$ et un deuxième argument $q$, plus fort que $p$, qui dirige la conclusion vers le sens opposé non-r: il pleut mais je dois sortir

Meta, XXXV, 3, 1990 
b) les concessions logiques, où $q$ est une cause qui devrait empêcher la réalisation de $p$ mais qui n'a pas l'effet attendu: je sors bien qu' il pleuve. il dit:

J.-C. Chevalier souligne ce qu'ont en commun les deux types de concession quand

La concession n'est que le nom donné à une propriété reconnue commune à divers instruments linguistiques: celle de lever une incompatibilité théorique entre deux énoncés. De sorte que la concession n'a d'existence que métalinguistique ${ }^{2}$.

Notre perspective étant fondamentalement sémantico-pragmatique, et non grammaticale, nous prenons donc le terme dans le sens le plus large, car cela nous permet d'envisager le problème du transfert de cette opération du français vers l'espagnol dans une perspective plus générale.

La plupart des traductions espagnoles, comme nous l'avons dit, expriment toutes les opérations concessives au moyen d'un nombre très restreint de connecteurs. Les plus fréquents sont :

pero: le connecteur oppositif/concessif par excellence; aunque: exprime la concession logique; sin embargo (et moins souvent no obstante): employé comme synonyme des deux connecteurs précédents.

Il existe toutefois des exceptions. Dans de bonnes traductions, nous pouvons percevoir des efforts pour pénétrer dans les nuances argumentatives et transmettre ces rapports avec fidélité. Parfois le traducteur a procédé à un vrai décodage et découvert le sens exact du connecteur. Par exemple, dans la phrase suivante, J. Cortázar a rendu l'«archiconnecteur» et, polysémique par excellence, par la valeur concessive qu'il véhicule:

(...) que la nomenclature officielle me donnait pour ma mère, et qui était bien davantage 3 .

(...) que la nomenclatura oficial me asignaba por madre, aunque era mucho más que eso ${ }^{4}$.

Certains connecteurs concessifs français montrent des difficultés intéressantes quand il s'agit de les traduire en espagnol. En voici quelques-uns.

\section{QUAND MÊME}

D'après Moeschler et Spengler, c'est «un des marqueurs les plus déroutants ${ }^{5}$ ». Il semble aussi dérouter parfois les traducteurs qui «oublient» sa présence :

Bonne journée quand même... grommela Maigret en quittant la cuisine ${ }^{6}$.

«Buenos días!»— murmuró Maigret, saliendo de la cocina?.

L'espagnol ne possède pas de connecteur exactement équivalent à quand même, ce qui oblige, pour trouver un équivalent, à une analyse de la valeur pragmatique dans chaque contexte.

Dans certains cas, quand même montre une valeur nettement concessive. Il résout une contradiction et confère à l'énoncé $q$, qu'il introduit, le statut argumentatif de non- $r$, c'est-à-dire qu'il dirige l'argumentation vers une conclusion opposée à celle de l'énoncé précédent. Le connecteur espagnol sera alors: $\sin$ embargo. Cependant, très souvent, quand même a aussi une valeur de «rattrapage», c'est-à-dire qu'il reprend d'une façon anaphorique l'énoncé $p$. La traduction espagnole serait alors a pesar de todo, a pesar de ello, con todo..., où ello et todo, rendent cette valeur anaphorique :

Elle l'a reconnu quand même 8 !

¿Lo reconoció, a pesar de ello ${ }^{9}$ ? 
Quand même véhicule aussi, dans certains cas, une valeur de type conclusif; $q$ est alors un argument qui veut aller contre les arguments présentés précédemment et clore le discours sans donner la possibilité que $q$ soit contredit. L'espagnol doit chercher alors un connecteur de type conclusif : aún así, así y todo... Par exemple, à la fin d'un article sur le philosophe Alain, nous trouvons cette phrase:

Ce vertueux eut quand même, malgré les apparences, une vie privée. (L'Express, 12 avril 1985.)

La traduction serait :

Aún así,

Así y todo, y pese a las aparencias, este virtuoso tuvo una vida privada.

D'après Jayez ${ }^{10}$, l'effet de même dans ce connecteur est de produire l'image d'une sorte de hiérarchie argumentative. Cette hiérarchie est mieux perçue dans certaines constructions. Pour souligner en espagnol que $q$ est un argument moins fort qui n'arrive pas à invalider $p$, il faut employer des connecteurs du type: de todos modos, de todas formas... Par exemple :

Le gouvernement, naturellement, n'est pas prêt à suivre ce genre de conseil. Mais il a quand même commis une grosse erreur.

(...) De todos modos ha cometido un grave error.

Parfois ce connecteur introduit une structure diaphonique où une deuxième voix reprend et interprète $p$ dans une autre perspective. Cette reprise prend parfois la forme d'une sorte de surprise devant une norme ou une croyance qui a été transgressée. Par exemple, la surprise de voir un musulman membre du parti communiste donne lieu à cet énoncé :

Inattendue, quand même, cette déclaration d'un enseignant dont le père, «beg» (chef) local a été éxécuté en 1951: «Cette année, j'ai adhéré au Parti Communiste». Mais l'islam? «Je reste croyant...». (L'Express, 8 août 1986.)

Cette impression de surprise pourrait être rendue en espagnol par la locution no deja de ser + adjectif ajoutée au connecteur de todos modos:

No deja de ser de todos modos sorprendente esta declaración de un maestro cuyo padre, «beg» (jefe) local fue ejecutado en 1951 (...)

Ou bien le sens de quand même peut être rendu par la locution toute seule. Ainsi, ce texte, où le connecteur exprime la contradiction, perçue par le locuteur, entre le régime communiste chinois et la présence de petites entreprises privées dans ce pays:

Pour faciliter l'avènement du vrai socialisme, il faut un peu de capitalisme. Les petites entreprise privées auront la possibilité d'employer jusqu'à huit personnes. «C'est quand même audacieux...» (L'Express, 6 novembre 1987.)

En espagnol la traduction serait:

(...) No deja de ser atrevido (...)

On ne peut pas oublier non plus la valeur réprobative de ce connecteur, si souvent employé dans l'interaction orale et si difficile à traduire. Devant quelqu'un qui crache sur le trottoir un Français peut dire:

Quand même! 
Un Espagnol devrait dire:

¡Ya está bien!

¡Oiga Vd.!

;Vamos, hombre!

dont la valeur réprobative est équivalente.

MÊME SI

Le dictionnaire français-espagnol, espagnol-français, de Larousse ${ }^{11}$ donne aún cuando comme la seule traduction possible de ce connecteur concessif. Mais, en réalité, les possibilités sont beaucoup plus nombreuses.

Même si véhicule dans la plupart des cas une valeur concessive du type hypothétique, d'après la classification de R. Martin qui définit cette argumentation comme celle qui exprime:

la vérité de $q$ dans tous les mondes potentiels où $p$ est vrai ${ }^{12}$

c'est-à-dire, dans la phrase:

Pierre s'en ira, même si Sophie revient

p

q

si $p$ est vérifié dans le cas le moins favorable $q$, cela veut dire qu'il est vérifié quel que soit $q$. Pour véhiculer la même opération argumentative, l'espagnol possède le connecteur incluso si, aussi à valeur hypothétique :

(...) le Liège rococo, enfoncé comme toute I'Europe dans l'absolutisme et la douceur de vivre, coule des jours actifs et tranquilles même si les élégants «Chiroux» (...) se font de temps en temps conspuer par les «Grignoux» 13 .

(...) la Lieja rococó, hundida igual que toda Europa en el absolutismo y la vida regalada, conoce dias activos y relativemente tranquilos, incluso si los elegantes «Chiroux» (...) se ven abuchear de cuando en cuando por los «Grignoux» 14 .

Dans d'autres cas, la valeur de même si approche des concessives scalaires et la traduction peut avoir recours à ce genre de connecteur en espagnol: por más que..., por mucho que....

l'élection présidentielle contraint les Français, même $s$ 'ils sont las, à se redéfinir par rapport au seul clivage gauche-droite. (L'Express, 23 octobre 1987.)

la elección presidencial obliga a los franceses, por muy cansados que estén (...)

Même si peut aussi avoir la valeur d'une concessive simple et il sera traduit alors par une des plusieurs possibilités d'exprimer cette opération en espagnol :

La coexistence dans les territoires occupés, même si elle est souvent explosive, a changé certaines mentalités, au bout de vingt années. (L'Express, 4 décembre 1987.)

La coexistencia en los territorios ocupados, pese a ser frecuentemente explosiva, ha cambiado ciertas mentalidades (...)

OR

Ce connecteur, si l'on en croit les dictionnaires français-espagnols, ne peut être traduit que par la locution ahora bien qui a la même étymologie (hac hora). Cependant, l'emploi de ahora bien, dont l'usage restreint est mis en évidence dans le dictionnaire de María Moliner: 
«Ahora bien» equivale a «pero» en expresiones como «haz lo que quieras $15_{\text {» }}$

est devenu de moins en moins fréquent dans la langue écrite, de sorte que, très souvent, il faut chercher d'autres équivalences. Les traducteurs espagnols le remplacent assez fréquemment par le connecteur «passe-partout» pero:

Il s'attendait à une protestation véhémente, voire à une scène violente. $O r$, il se trouva devant un être abattu, sans réaction, qui ne prononça pas un mot ${ }^{16}$.

Esperaba encontrarse con una vehemente protesta, incluso una violenta escena. Pero se halló ante un ser abatido, sin reacción, que no pronunciaba una palabra ${ }^{17}$.

Le Petit Robert définit or comme : ment $^{18}$

- une conjonction marquant un moment particulier d'une durée ou d'un raisonne-

En disant que $\ll p$ or $q »$, le locuteur veut montrer que $q$ ajoute à $p$ des éléments nouveaux et inattendus et, par conséquent, met en question la vérité de $p$. Le schéma argumentatif de ce connecteur, assez proche de celui indiqué par C. Sirdar-Iskandar ${ }^{19}$ à propos de eh bien!, montre une suite inattendue $q$. Par l'effet de ses éléments nouveaux, cette suite inattendue transforme la conclusion $r$ en une conclusion $r$ '. Dans l'exemple suivant :

Les Japonais partagent avec les Américains la royauté du monde électronique : or la guerre des étoiles, c'est d'abord des capacités de communications et de calculs ultra-rapides. (L'Express, 12 avril 1985.)

le traducteur espagnol doit faire appel à un connecteur qui souligne que «la guerre des étoiles» introduit dans $q$ un élément nouveau qui change la perspective pacifiste de $p$ pour transporter l'argumentation au domaine de la guerre nucléaire. Ce tournant de l'argumentation peut être rendu en espagnol par:

pues bien...

ou même :

pero resulla que...

qui reprend cette nuance inattendue. Parfois la longueur de cette deuxième locution vis-àvis du phonème atone or fait refuser cette solution. Mais quand or est suivi de voilà, la traduction par pero resulta que est très adéquate, comme dans cette phrase :

Les impôts, les prix, le dollar, tout baissait. Ce qui amenait la plupart des spécialistes à prédire de nouveaux succès à l'Administration Reagan. Or voilà que l'on découvre brusquement que le paysage a changé. (L'Express, 15 août 1986.)

(...) Pero resulta que de repente el paisaje ha cambiado.

Dans ce genre d'argumentations, $q$ peut aussi introduire des éléments tout à fait opposés à ceux de $p$. On peut alors avoir recours à des connecteurs espagnols qui marquent nettement l'opposition, tels que : en cambio, sin embargo. Dans cette phrase:

Le coût de fabrication de la machine était très élevé, et, au Japon les banques ne prêtent qu'aux riches. $O r$, je n'avais pas un sou. (L'Express, 3 avril 1987.)

donnerait une traduction du type :

Yo, en cambio, no tenía un duro.

Yo, sin embargo, no tenia un duro. 
Dans d'autres cas, $q$ nie absolument la vérité de $p$ :

Aux premiers jours de la guerre, l'Amérique a cru que l'Irak balaierait les troupes khoméinistes. Or il n'en est rien...

la traduction demanderait aussi un connecteur d'opposition :

(...) $Y$ en cambio no ocurrió así (...)

$q$ peut introduire un élément de correction ou de précision de la vérité de $p$, mais sans montrer une opposition absolue :

On accuse souvent la Communauté de bureaucratie. $O_{r}$, la principale bureaucratie est celle que le Conseil a imposée depuis vingt-cinq ans à la Commission en la flanquant de comités (M. J. Delors au Parlement européen.)

Or serait rendu alors en espagnol par un connecteur du type : sin embargo, no obstante...

\section{POURTANT}

Généralement, dans «p pourtant $\mathrm{q} »$, la force argumentative de $q$ corrige, modifie, s'oppose ou invalide l'énoncé $p$ et change le sens de la conclusion. Dans la traduction de ces argumentations, pourtant est rendu en espagnol par sin embargo, no obstante. Mais, la valeur anaphorique ou de «rattrapage» que nous avons remarquée dans le connecteur quand même y est aussi présente assez souvent. La traduction devrait alors essayer de garder cette nuance:

Il n'est pas plaisant de trouver une ordonnance dans son assiette. Et pourtant, de plus en plus, la médecine s'immisce dans l'alimentation de l'homme.(L'Express, 25 mars 1988.)

No es atractivo encontrarse una receta en el plato. $\mathrm{Y}$ con todo, la medicina se inmiscuye cada vez más en la alimentación humana. (Traduction apparue dans El País, 28 mars 1988.)

Ce connecteur peut laisser aussi l'énoncé $q$ implicite. Sa valeur est alors d'inverser complètement le sens de la direction argumentative de $p$ :

Aux yeux des Français de 1987, le nylon, c'est mochard et craquant (...). Trop banal pour qu'on se souvienne qu'il fut un mythe. Et pourtant! (L'Express, 27 novembre 1987.)

Cela donnerait en espagnol:

(...) demasiado banal para que nadie se acuerde de que fue un mito. $\mathrm{j} Y$ vaya si lo fue! lisons:

Dans d'autres cas c'est $p$ qui reste implicite. Dans un texte sur les drogues nous

Valérie n'a ni le regard brillant ni les pupilles fixes et sa mère n'a jamais rien remarqué. (...)

Déjà, sa jeune sœur Caroline, 15 ans, suit l'exemple de Valérie: «Pourtant, au début je ne voulais pas qu'elle fume. Mais elle s'est mise à respirer du détachant...» (L'Express, 3 octobre 1986.)

En espagnol le connecteur serait y eso que, qui permet de reprendre un $p$, implicite, au moyen du pronom eso:

«Y eso que, al principio, no quería fumase (...)» 


\section{D'AUTRES FORMULES CONCESSIVES FRANÇAISES}

Il faut tout d'abord remarquer que la formule concessive par excellence «p bien que q» ne peut être construite en français qu'avec le verbe au subjonctif. La construction espagnole admet aussi l'indicatif:

1. salgo aunque llueve

2. salgo aunque llueva

où 1. montre l'existence réelle de l'empêchement et 2 . exprime un empêchement hypothétique.

Le connecteur aunque - équivalent de bien que - est plus employé que ce dernier et occupe une partie du champ sémantique de mais et de pourtant. Aunque a un synonyme, aún, qui comme J.-C. Chevalier, M. Launay et M. Molho le démontrent ${ }^{20}$, en plus de son sens concessif, partage aussi son champ sémantique avec encore, toujours et même.

Aún diffère de aunque en ce que l'argument introduit par lui ne peut pas présenter un verbe à la forme personnelle:

Bien que la flotte soit vieille, les accidents ne sont pas dus à des avaries. (L'Express, 8 août 1986.)

Aunque la flota es vieja (...) Aún siendo vieja la flota (...)

Le français possède aussi quelques constructions concessives où le connecteur a été remplacé par une locution verbale. L'espagnol n'a pas de formules équivalentes.

Ainsi, l'expression avoir beau peut être transférée en espagnol par un connecteur de concession simple (aunque, sin embargo...) ou aussi, très souvent, par un connecteur du type scalaire:

Les sceurs de Fernande, (...) avaient beau lui dire qu'on aime déjà l'enfant qui va naitre, elle ne parvenait pas à établir un rapport entre ses nausées (...) et la petite créature 21 .

Por mucho que las hermanas de Fernande [...] le dijesen que se quiere de antemano al niño que va a nacer, no conseguía establecer una relación entre sus naúseas [...] y la pequeña criatura $^{22}$

Avoir beau est aussi employé dans des expressions plus ou moins lexicalisées en francais qui doivent être traduites par des formules semblables:

Ils protestent contre une annonce qu'ils jugent dangeureuse. Ils auront beau faire : la littérature médicale est définitivement tombée dans le domaine public. (L'Express, 11 mars 1988.)

Protestan contra un anuncio que consideran peligroso. Aunque es inútil, pues la literatura médica ha entrado definitivamente en el dominio público.

La formule «imparfait du subjonctif du verbe être + pronom sujet» (fût-ce, fussent-ils...) peut être traduite par un énoncé sans verbe à cause de la valeur copulative d'être:

Les drogues, fussent-elles dures, ne parviennent pas toujours à vaincre la douleur. (L'Express, 15 janvier 1988.)

Las drogas, aún las duras, no consiguen siempre vencer el dolor.

Le conditionnel du verbe être placé dans une proposition négative peut aussi donner lieu à une concession sans connecteur :

Ce livre fera sûrement grand bruit. Ne serait-ce que parce qu'il ouvre la campagne électorale. (L'Express, 22 janvier 1988.) 
L'espagnol a besoin d'une conjonction concessive:

(...) aunque sólo sea porque abre la campaña electoral.

Le subjonctif négatif peut véhiculer aussi une argumentation concessive:

N'en déplaise aux politicologues, une nouvelle culture est née.

qui peut être rendue en espagnol soit par un connecteur simple:

(...) aunque no les guste a los politicólogos (...)

soit, et parfois avec priorité, par une formule du type scalaire :

Les guste o no a los politicólogos (...)

Les constructions concessives sont complexes et la comparaison entre deux langues montre l'abondance de moyens d'expression de ce type dont elles disposent. Il reste encore beaucoup de différences remarquables entre le français et l'espagnol concernant cette opération argumentative.

Les possibilités de traduction en espagnol du connecteur mais mériteraient tout un article, les constructions dont l'espagnol dispose pour exprimer les formules concessives scalaires et extensionnelles diffèrent beaucoup des françaises, les formules concessives avec participe présent du français n'ont pas d'équivalent exact en espagnol...

Les enchaînements argumentatifs jouent un rôle clé dans la sémantique d'un texte et notre but était de signaler qu'on ne peut pas laisser de côté la valeur de ces morphèmes, car toutes leurs petites nuances sémantiques ont un poids dans le texte que la traduction doit garder.

Les moyens d'articulation des relations argumentatives d'une langue sont toujours d'une grande richesse. Une analyse de ces relations dans le texte de départ et l'étude de l'éventail de possibilités pour reproduire ces moyens dans la langue d'arrivée pourraient faire avancer la pratique traduisante et, en même temps, la comparaison de la façon d'argumenter à partir de différents systèmes linguistiques apporterait, peut-être, des éléments de réflexion nouveaux à la théorie de la traduction.

\section{NOTES :}

1. DUCROT, O. et J.-C. ANSCOMBRE (1977), «Deux mais en français ?», Lingua, no 43, pp. 23-40.

2. CHEVALIER J.-C., M. LAUNAY, M. MOLHO (1983), «L'expression de la concession en espagnol (Le signifiant aún/aunque), L'expression de la concession», Actes du Colloque tenu les 3 et 4 décembre 1982 par le Département de linguistique de l'Université de Paris-Sorbonne, Paris, p. 71

3. YOURCENAR, M. (1974), Mémoires d'Hadrien, Gallimard, p. 174.

4. YOURCENAR, M. (1982), Memorias de Adriano (traducción de Julio Cortázar), Edhasa, p. 183.

5. MOESCHLER J., N. de SPLENGLER (1981), «Quand même: de la concession à la réfutation. Les différents types de marqueurs et la détermination des fonctions des actes de langage en contexte», Actes du 1 er Colloque de pragmatique de Genève, Cahiers de linguistique française, $\mathrm{n}^{\circ} 2$, Université de Genève, Genève, p. 83 .

6. SIMENON, G. (1968), Maigret à Vichy, Paris, Presses de la Cité, p. 92.

7. SIMENON, G. (1978), Maigret en Vichy, Novelas de Maigret (traducción de L. Hernández Alonzo), Aguilar, tome X, p. 95.

8. Maigret à Vichy, p. 42

9. Maigret en Vichy, p. 49

10. JAYEZ J.(1982), «Quand bien même pourtant, Pourtant quand même, Concession et Consécution dans le discours», Cahiers de linguistique française, no 4, Université de Genève, Genève, p. 213.

11. GARCIA-PELAYO, R., J. TESTAS (1967), Dictionnaire moderne français-espagnol, espagnol-français, Paris, Larousse, p. 466. 
12. MARTIN, R. (1987), Langage et croyance. Les "univers de la croyance» dans la théorie sémantique, Bruxelles, Pierre Mardaga éditeur, p. 87.

13. YOURCENAR, M. (1974), Souvenirs pieux, Paris, Gallimard, coll. «Folio», p. 82

14. YOURCENAR, M. (1974), Recordatorios (traducción de E. Calatayud), Ediciones Alfaguara, p. 84

15. MOLINER, M.( 1983), Diccionario del uso del español, Madrid, Gredos, p. 100.

16. Maigret à Vichy, p. 182

17. Maigret en Vichy, p. 178-179

18. Petit Robert I, Dictionnaire alphabétique et analogique de la langue française, Paris, 1977, p. 1317

19. SIRDAR-ISKANDAR, C.(1980), «Eh bien! le Russe a donné cent francs», dans O. Ducrot et al, Les Mots du discours, Paris, éditions de Minuit, p. 161-191.

20. CHEVALIER J.-C., M. LAUNAY, M. MOLHO, «De la concession en espagnol...», p. 62.

21. YOURCENAR, M., Souvenirs pieux, p. 27

22. YOURCENAR, M., Recordatorios, p. $\mathbf{3 0}$

(Les traductions des exemples tirés de L'Express sont miennes.) 